In Crescendo, 2019; 10(2): 349-362

Fecha de recepción: 30/01/2019

Fecha de aceptación: 06/06/2019

\title{
EL SOFTWARE JCLIC EN LAS OPERACIONES BÁSICAS CON LOS NÚMEROS NATURALES BAJO EL MODELO DEL APRENDIZAJE BASADO EN PROBLEMAS
}

\author{
THE JCLIC SOFTWARE IN BASIC OPERATIONS WITH \\ NATURAL NUMBERS UNDER THE MODEL OF \\ PROBLEM-BASED LEARNING
}

Eliana N. Bautista ${ }^{1}$, Lennis Santafe ${ }^{2}$

\section{RESUMEN}

La presente Investigación es de tipo cualitativa, la población se delimitó por 150 por estudiantes del Centro Educativo Rural los Guayabales del Municipio de Pamplonita y como muestra a 7 estudiantes del grado 3 o Centro Educativo Rural los Guayabales, el objetivo fue analizar el proceso de aprendizaje de las operaciones básicas con los números naturales en el marco del modelo del aprendizaje basado en problemas empleando el software Jclic en los estudiantes del grado tercero del Centro Educativo Rural los Guayabales del Municipio de Pamplonita, la mediación se desarrolló por medio de talleres en tres fases: parte teórica conceptual y ejercitación en hojas de papel; desarrollo del trabajo por medio del software Jclic desde lo teórico y posteriormente desde lo práctico; refuerzo y desarrollo en casa. como resultado de la entrevista aplicada a los estudiantes, se puede afirmar que los estudiantes en mención asumen las clases de matemáticas como muy importantes para su desarrollo académico, social y laboral, sin

1 Estudiante Maestría en Educación. km 1 vía Bucaramanga, Colombia, elianitabautista@hotmail.com

$2 \mathrm{PhD}$ en Educación. Docente Universidad de Pamplona. km 1 vía Bucaramanga, Colombia, lenis.santaf7@gmail.com 
embargo, las perciben monótonas y poco dinámicas, expresando que quisieran que estas fueran diferentes. De la misma manera, expresan que sus malos resultados en la asignatura se relacionan con la dificultad para entender los conceptos y procedimientos, así como por la falta de interés por estudiar y hacer los trabajos en casa.

PALABRAS CLAVE: Operaciones básicas con números naturales, aprendizaje basado en problemas, software Jclic, test SABER.

\section{ABSTRACT}

This research is qualitative, the population was delimited by 150 students from the Guayabal Rural Educational Center of the Municipality of Pamplonita and as a sample of 7 students from the 3rd degree Rural Educational Center los Guayabales, the objective was to analyze the learning process of the basic operations with the natural numbers in the framework of the problem-based learning model using the Jclic software in the third grade students of the Guayabales Rural Educational Center of the Municipality of Pamplonita. The mediation was developed through workshops in three phases: conceptual theoretical part and exercise in sheets of paper; development of work through the Jclic software from the theoretical and later from the practical; reinforcement and development at home. As a result of the interview applied to the students, it can be affirmed that the students in question assume the mathematics classes as very important for their academic, social and work development, however, they perceive them as monotonous and not very dynamic, expressing that they would like these they were different. In the same way, they express that their poor results in the subject are related to the difficulty in understanding the concepts and procedures, as well as the lack of interest in studying and doing homework.

KEY WORDS: basic operations with natural numbers, Problem Based Learning, Jclic software, SABER tests.

\section{INTRODUCCIÓN}

Teniendo en cuenta que los estudiantes del grado tercer Centro Educativo Rural Los Guayabales del Municipio de Pamplonita, presentan dificultades para desarrollar las operaciones básicas en matemáticas, y que dicha situación incide en su desempeño académico, es preciso citar a (Jimeno, 2002), quien relaciona el bajo rendimiento académico en matemáticas con dificultades de aprendizaje relacionadas con déficits sensoriales, motores, retraso mental, trastornos emocionales, deprivación sociocultural u otros.

De los postulados del autor citado, se destaca, para este caso, la depravación sociocultural, toda vez que en entornos rurales se percibe la tendencia a imitar 
modelos de comportamiento propios de culturas urbanas, lo cual ocasiona conflictos de identidad en los estudiantes, afectando directamente su desempeño académico y su comportamiento en su contexto.

En lo referente a los docentes, (Carrillo \& Ríos, 2009), expresan que el bajo rendimiento académico en matemáticas puede estar dado por la escasa planificación de estrategias y por el incorrecto uso de estrategias metodológicas. Señalan, además, que se evidencia falta de atención frente a las diferencias individuales para satisfacer las necesidades de cada estudiante y que no existe asociación directa entre teoría y práctica, por lo cual el estudiante no aprecia ninguna correlación con las realidades concretas.

De lo anterior se infiere la gran responsabilidad de los docentes en el proceso educativo, específicamente en el aprendizaje de sus estudiantes, toda vez que dichas actividades requieren de su proactividad, ingenio y dedicación, lo cual ha de plasmar en estrategias didácticas concretas, planificadas según las necesidades propias de sus estudiantes y contextualizadas a la realidad que viven estos en sus entornos familiares sociales y académicos.

En la misma línea, (Abrate, Raquel, Pochulu, \& Vargas, 2006), enuncian que las dificultades asociadas a los procesos de enseñanza están relacionadas con la institución escolar, con el currículo de Matemática y con los métodos de enseñanza. Desde esta perspectiva, los autores sugieren que los métodos de enseñanza deben estar ligados tanto a los elementos organizativos de la institución escolar, como a la organización curricular.

De acuerdo con los planteamientos anteriores, se infiere la necesidad de plantear un currículo contextualizado a la realidad y a las necesidades educativas de los estudiantes, realizando las adaptaciones necesarias para su pleno desarrollo. En este sentido, las directivas de las instituciones tienen una gran responsabilidad en el diseño de un currículo flexible y ajustado a su propio contexto.

Desde otra perspectiva, es procedente mencionar que según el Ministerio de Educación Nacional (MEN, 2016) en las pruebas PISA 2015 Colombia obtuvo 390 puntos, lo cual representa una mejora de 20 puntos en comparación al 2006. No obstante, el $66 \%$ de los estudiantes colombianos que participaron de las pruebas poseen bajo rendimiento en matemática.

En lo referente a los resultados obtenidos por los estudiantes, en el ámbito nacional el (MEN, 2018), expresa que para los grados 3, 5 y 9 el puntaje promedio 
obtenido en 2017 en matemáticas fue considerablemente menor al presentado en 2016.

Los resultados obtenidos por los estudiantes del Centro Educativo Rural los Guayabales del Municipio de Pamplonita en las últimas pruebas externas, justifican en su totalidad el desarrollo de la presente investigación, toda vez que es necesario fortalecer el proceso de aprendizaje de las matemáticas, y específicamente en lo que hace referencia a las cuatro operaciones básicas, es decir, suma, resta, multiplicación y división.

En tal sentido, es imprescindible identificar e implementar estrategias conducentes al mejoramiento de los mencionados indicadores, ya que estos se constituyen en referentes para determinar la calidad de los procesos educativos en las diferentes instituciones.

Por otra parte, la presente investigación cobra gran relevancia desde la perspectiva de la utilización de los recursos tecnológicos con los que cuenta la Institución Educativa, pues a partir de ellos, los estudiantes se muestran más interesados y son más receptivos a este tipo de metodologías, máxime cuando se trata de herramientas novedosas y facilitadoras de su proceso de aprendizaje. Por lo tanto, (López, 2013), expresa que, con el uso de la tecnología, los estudiantes aprenden con menos tiempo, tienen acceso a múltiples recursos educativos y a entornos de aprendizaje, existe personalización del proceso de enseñanza y aprendizaje y hay más contacto y colaboración entre compañeros.

Desde la perspectiva de los docentes, la utilización de medios tecnológicos se convierte en fuente de recursos educativos para la enseñanza, hay mayor contacto con los estudiantes, lo libera de los trabajos repetitivos y monótonos, facilita la evaluación y se evidencia una continua actualización profesional (López, 2013). Desde el proceso de aprendizaje, el uso de recursos tecnológicos incrementa el interés y la motivación, se incrementa la actividad intelectual, se desarrolla la iniciativa, hay mayor comunicación entre los profesores y los alumnos y se evidencia el aprendizaje cooperativo (López, 2013).

Otro aspecto a tener en cuenta desde los aspectos que justifican la investigación, es el relacionado directamente con el desarrollo de las competencias en matemáticas, ya que por medio de la comprensión, desarrollo y puesta en práctica de las operaciones básicas, los estudiantes adquieren la habilidad para interpretar y expresar con claridad y precisión informaciones, datos y argumentaciones, lo 
que aumenta la posibilidad real de seguir aprendiendo a lo largo de la vida; se logra la disposición favorable y progresiva seguridad y confianza hacia la información y las situaciones que contienen elementos o soportes matemáticos, así como hacia su utilización cuando la situación lo aconseja, basadas en el respeto y el gusto por la certeza y en su búsqueda a través del razonamiento (Euskadi, 2014).

Desde la perspectiva del Aprendizaje Basado en Problemas (ABP), se puede mencionar que en los métodos actuales de enseñanza no se aplica tal, es decir, pues no se parte de un problema identificando las necesidades de aprendizaje, sino que por el contrario se aplica el proceso convencional, lo cual para los estudiantes resulta monótono y resta interés a los temas tratados; se puede inferir que el fortalecimiento en el manejo de las operaciones básicas en matemáticas habrá de fortalecer los procesos de aprendizaje en los estudiantes del grado tercero del Centro Educativo Rural los Guayabales del Municipio de Pamplonita y que su verdadero impacto se verá reflejado en las pruebas internas y externas que estos presenten, redundando en su desempeño académico.

El objetivo fue analizar el proceso de aprendizaje de las operaciones básicas con los números naturales en el marco del modelo del aprendizaje basado en problemas empleando el software Jclic en los estudiantes del grado tercero del Centro Educativo Rural los Guayabales del Municipio de Pamplonita.

\section{El aprendizaje}

El aprendizaje según DIGECADE (2010), se define como "el proceso por el cual las personas adquieren cambios en su comportamiento, mejoran sus actuaciones, reorganizan su pensamiento o descubren nuevas maneras de comportamiento y nuevos conceptos e información". Es privilegio del y de la docente orientar el aprendizaje en tres terrenos o categorías principales, las cuales se sintetizan en: Aprendizaje de los saberes y su aplicación; Aprendizaje de habilidades y destrezas; Aprendizaje de valores y actitudes.

Las anteriores categorías coinciden con los pilares del conocimiento propuestos por Jacques Delors quien los percibe como aprendizajes fundamentales en el transcurso de la vida de cada persona y como las bases de las competencias del futuro. Delors propuso cuatro pilares: Aprender a conocer, aprender a hacer, aprender a ser, aprender a vivir juntos. Más tarde la Oficina Regional de Educación para América Latina y el Caribe (PRELAC) propuso agregar como quinto pilar del conocimiento "Aprender a emprender". 


\section{Aprendizaje Basado en Problemas}

El aprendizaje Basado en Problemas (ABP), es entendido como una estrategia de enseñanza-aprendizaje en la que la adquisición de conocimientos y el desarrollo de habilidades y actitudes resultan de gran importancia.

En el ABP un grupo de estudiantes se reúne, con la facilitación de un tutor, a analizar y resolver un problema seleccionado o diseñado especialmente para el logro de ciertos objetivos de aprendizaje. Durante el proceso de interacción de los estudiantes para entender y resolver el problema se logra, además del aprendizaje del conocimiento propio de la materia, que puedan elaborar un diagnóstico de sus propias necesidades de aprendizaje, que comprendan la importancia de trabajar colaborativamente, que desarrollen habilidades de análisis y síntesis de información, además de comprometerse con su proceso de aprendizaje (Instituto Tecnológico de Monterrey, 2002), citado por (Gélvez, 2017)

El sustento teórico del ABP se encuentra en los siguientes principios básicos:

- El entendimiento con respecto a una situación de la realidad surge de las interacciones con el medio ambiente.

- El conflicto cognitivo al enfrentar cada nueva situación estimula el aprendizaje.

- El conocimiento se desarrolla mediante el reconocimiento y aceptación de los procesos sociales y de la evaluación de las diferentes interpretaciones individuales del mismo fenómeno.

Por otra parte, el ABP incluye el desarrollo del pensamiento crítico en el mismo proceso de enseñanza - aprendizaje, no lo incorpora como algo adicional, sino que es parte del mismo proceso de interacción para aprender, además, el ABP busca que el alumno comprenda y profundice adecuadamente en la respuesta a los problemas que se usan para aprender abordando aspectos de orden filosófico, sociológico, psicológico, histórico, práctico, etc. (Instituto Tecnológico de Monterrey, 2002), citado por (Gélvez, 2017)

\section{Las operaciones básicas con Número Naturales}

Las cuatro operaciones básicas de matemática son fundamentales para la vida futura y cotidiana de los niños y las niñas, las operaciones de esta naturaleza pierden valor e importancia cuando se exageran en el número de operaciones a realizar por parte de las y los alumnos, por lo consiguiente, se vuelve mecanizado. 
La capacidad para producir rápida y eficazmente una suma o producto de dos dígitos simples enteros es una de las principales habilidades que un niño debe adquirir en los primeros años de la escuela. Dos décadas de investigaciones en procesos básicos subyacentes a esta simple tarea han mostrado que los niños gradualmente van avanzando desde el uso de un amplio número de algoritmos para el desempeño en la aritmética simple hasta confiar fundamentalmente en las repercusiones directas de las respuestas conocidas (Roncal \& Cabrera, 2002).

Para abordar cada una de las operaciones básicas, se empezará por decir que la suma es una operación básica por su naturalidad, que se representa con el signo $(+)$, y consiste en combinar o añadir dos números o más para obtener una cantidad final o total (Masa, 2014). Al respecto, (Roncal \& Cabrera, 2002), mencionan que algunos de los errores más comunes en la suma son: contar para hallar la suma, añadir el número que se lleva al final, olvidarse de añadir el número que se lleva, reiniciar la suma parcialmente hecha y agregar irregularmente el $n$ mero que se lleva, entre otros.

La resta, por su parte, según (Masa, 2014), se trata de una operación de descomposición que consiste en, dada cierta cantidad, eliminar una parte de ella. El resultado se conoce como diferencia o resto, lo anterior es complementado por (Roncal \& Cabrera, 2002), mencionado que los errores más comunes en esta operación son: contar para hallar la resta, errores debidos a ceros en el minuendo, nombrar los términos al revés, restar el minuendo del sustraendo, poner cero cuando la cifra del sustraendo es superior a su correspondiente en el minuendo y restar dos veces de la misma cifra del minuendo, entre otros.

Referente a la multiplicación (Masa, 2014), menciona que es una operación matemática que consiste en sumar un número tantas veces como indica otro número. (Roncal \& Cabrera, 2002), mencionan además que los errores más comunes en esta operación son: olvidarse de llevar, escribir el número que se lleva, errores al agregar el número que se lleva a cero, multiplicar el número que se lleva, agregar dos veces el número que se lleva y agregar un número cuando no se lleva.

Por último, la división, según (Masa, 2014), es una operación de descomposición que consiste en averiguar cuántas veces un número (divisor) está contenido en otro número (dividendo). El resultado de una división recibe el nombre de cociente. De manera general puede decirse que la división es la operación inversa de la multiplicación. Al respecto, (Roncal \& Cabrera, 2002), mencionan que los 
errores más comunes de los estudiantes al realizar una división son: hallar un resto superior al divisor, hallar el cociente por sucesivas multiplicaciones, olvidar el resto al seguir dividiendo, omitir el cero en el cociente, omitir una cifra de dividendo, y contar para hallar el cociente, entre otros.

\section{Papel de las TIC en la innovación educativa}

Las TIC, además de ser herramientas tecnológicas, hacen parte del ambiente innovador que hoy día se vive en la educación y en el aprendizaje, es de suma importancia no pensar que solo las TIC hacen parte de este ambiente innovador, por el contrario, optar por otros aspectos que están inmersos en este medio, tales como el desarrollo del pensamiento crítico, autónomo y creativo mediante el trabajo en equipo, entre otros muchos aspectos que día a día ayudan a mejorar el proceso enseñanza-aprendizaje a través de la innovación.

La innovación involucra también a aquella generación de ideas que pueden ocasionar mejoras en los procesos educativos, pero esta no está necesariamente vinculada con algún tipo de tecnología. Teniendo en cuenta la reciente digitalización del mundo, producto del desarrollo y popularización del computador y el Internet, ha cambiado el modelo de distribución de la información y ha dado lugar a la Sociedad del Conocimiento donde las ideas y sus aplicaciones cobran cada vez más valor y las interconexiones entre lugares, personas, economías y disciplinas se hacen cada vez más evidentes.

\section{Desarrollo de competencias matemáticas con uso de tecnología}

En el estudio de las matemáticas el término competencia matemática se refiere a las capacidades de los estudiantes para analizar, razonar y comunicar eficazmente el proceso de resolución de problemas matemáticos que se presenten en una variedad situaciones (INECSE, 2005). El proceso de resolución de un problema incluye diferentes fases entre las que se encuentran: a) Identificar las variables presentes en el problema; b) Representar el problema en forma diferente; c) Establecer relaciones entre las variables del problema; d) Establecer relaciones entre las representaciones empleadas; e) Identificar las matemáticas que pueden ser relevantes para la solución del problema; f) Relacionar el problema con otro más simple; g) Utilizar un modelo matemático para representar el problema; h) Justificar los resultados y i) Comunicar el proceso y la solución (García \& Benítez, 2011). 
En aras de desarrollar plenamente las competencias matemáticas, en los últimos años se ha incluido el uso de las TIC en el proceso educativo, toda vez que se considera que estas facilitan la realización de múltiples trabajos en la comunidad educativa: gestión de las instituciones, elaboración de materiales didácticos específicos, como instrumentos de apoyo pedagógico, etc.; contribuyen para mejorar los procesos de enseñanza y aprendizaje, ofrecen nuevas posibilidades para la innovación educativa, para desarrollar nuevos entornos de aprendizaje virtual, sistemas de tele formación, que contribuyen a superar las limitaciones geográficas que imponen los sistemas educativos presenciales. Se han establecido categorías para evaluar las competencias con que cuenta una persona para realizar tareas en forma efectiva en un ambiente digital; estas competencias se agrupan en bloques tales como el conocimiento de los sistemas informáticos (hardware, redes, software); el uso básico del sistema operativo; la comunicación interpersonal y trabajo colaborativo en redes; el procesamiento de textos; tratamiento de la imagen y actitudes generales ante las TIC (ICEC, 2004).

\section{Software JCLIC para proceso de aprendizaje}

JClic es un entorno para la creación, realización y evaluación de actividades educativas multimedia, desarrollado en la plataforma Java. Es una aplicación de software libre basada en estándares abiertos que funciona en diversos entornos operativos: Linux, Mac OS X, Windows y Solaris. JClic se constituye en una herramienta de apoyo durante el proceso de enseñanza y aprendizaje, toda vez que permite que los estudiantes aprendan jugando, se encuentren motivados e interesados. De la misma manera promueve la participación constante durante las sesiones de aprendizaje, logrando que los estudiantes desarrollen diferentes capacidades como la observación, identificación, comparación, clasificación, discriminación, análisis, organización y argumentación, entre otras (Zonaclic, 2008).

\section{MÉTODOS}

La presente investigación es de tipo cualitativo, el cual es definido y descrito por (Taylor \& Bogdan, 1987), citados por (Balcazar, González, \& Gurrola, 2013) como la investigación que produce datos descriptivos: las propias palabras de las personas, habladas o escritas, y la conducta observable.

La investigación cualitativa es de tipo inductivo, donde los investigadores desarrollan conceptos, intelecciones y comprensiones partiendo de pautas de los 
datos, y no recogiendo datos para evaluar modelos, hipótesis o teorías preconcebidas. En este tipo de estudio los investigadores siguen un diseño de la investigación flexible y comienzan sus estudios con interrogantes vagamente formulados (Quecedo \& Castaño, 2003), citados por (Peñaranda \& Pérez, 2017).

Por otra parte, el enfoque es de tipo investigación - acción, respecto de la cual (Elliot, 2000), citado por (Moreno, 2017) expresa que consiste en hacer una reflexión sobre las prácticas impartidas por los docentes en el aula con el fin de detectar falencias y tomar decisiones que contribuyan a la corrección y no solo realizar tipos de investigación que no permiten reflexionar sobre nuestras propias decisiones. Dando a entender que no deben ser absolutas, sino que pueden ser objeto de cambio. Cambio que debe resultar de la propia reflexión.

\section{Población y Muestra}

La población está conformada 150 por estudiantes del Centro Educativo Rural los Guayabales del Municipio de Pamplonita.

De las características de la población se puede mencionar el que 37\% presenta altos índices de desnutrición; el 62\% se dedica a labores de agricultura, minería y ganadería, y el $1 \%$ son empleados o comerciantes. En cuanto a la composición familiar, el 55\% son hogares funcionales, en el 18\%falta alguno de los padres, en el $12 \%$ viven personas diferentes al núcleo familiar y el $5 \%$ son hogares de segundas nupcias o unión libre.

Por su parte, la muestra está conformada por 7 estudiantes del grado 3ํㅡentro Educativo Rural los Guayabales del Municipio de Pamplonita.

\section{Instrumentos para la Recolección}

La recolección de la información es un proceso que requiere técnicas e instrumentos con un propósito específico, para lo cual se encuentran una gran variedad de enfoques tanto cuantitativos como cualitativos que se pueden aplicar y que dependen del tipo de investigación. (Hernández, Fernández, \& Baptista, 2010).

Para la recolección de información, y teniendo en cuenta el planteamiento de los objetivos de la investigación, se utilizarán los siguientes instrumentos:

Instrumento № 1 Entrevista a estudiantes: cuestionario aplicado a estudiantes con el fin de conocer sus intereses y expectativas frente al área de matemáticas. 
Instrumento № 2 Evaluación Diagnóstica: consistió en una prueba de saberes específicos que, de acuerdo con los estándares básicos de competencias, deben tener los estudiantes en el grado tercero.

Instrumento №3 Diario de Campo: es registro del desarrollo da cada una de las actividades a ejecutar. Es un instrumento utilizado por los investigadores para registrar aquellos hechos que son susceptibles de ser interpretados. En este sentido, el diario de campo es una herramienta que permite sistematizar las experiencias para luego analizar los resultados (Universidad Tecnológica de Pereira, 2017). Para este caso el formato fue utilizado para recoger las impresiones del docente durante la aplicación de cada una de las actividades.

Instrumento №4 Prueba de salida: evaluación final aplicada a los estudiantes, con el fin de determinar la efectividad de la estrategia aplicada.

\section{Validación de los Instrumentos}

Con el fin de dar crédito a la información recolectada, se hace necesario que los instrumentos para la recolección de información sean validados por expertos en investigación y el área específica, por tal razón, los instrumentos utilizados fueron validados por la Doctora Leny Santafé, pares evaluadores de la Universidad de Pamplona y pares académicos de CER Guayabales.

\section{RESULTADOS}

Respecto a la entrevista aplicada a los estudiantes, se puede afirmar que los estudiantes en mención asumen las clases de matemáticas como muy importantes para su desarrollo académico, social y laboral, sin embargo, las perciben monótonas y poco dinámicas, expresando que quisieran que estas fueran diferentes. De la misma manera, expresan que sus malos resultados en la asignatura se relacionan con la dificultad para entender los conceptos y procedimientos, así como por la falta de interés por estudiar y hacer los trabajos en casa.

Por su parte, en el desarrollo de cada uno de los talleres, los estudiantes se mostraron muy motivados debido a la metodología empleada, pues se iniciaba con actividades de reflexión y desarrollo del pensamiento, luego se hacía una explicación del tema, para seguidamente pasar al trabajo directo con el software Jclic, y por último un refuerzo y actividades en casa.

Con la propuesta de este proyecto se busca fortalecer el proceso de aprendi- 
zaje de las operaciones básicas con los números naturales en los estudiantes del grado tercero del centro educativo rural los guayabales del municipio de pamplonita, mediante la implementación del software Jclic, bajo el modelo del aprendizaje basado en problemas.

De acuerdo con lo anterior, la solución propuesta a la problemática descrita se basa en una estrategia didáctica por medio de talleres que los estudiantes resolvieron en clase, e incluso en la casa, los cuales estuvieron organizados así: Talleres 1 y 2 enfocados al desarrollo de la suma y sus propiedades. Talleres 3 y 4 enfocados al desarrollo de la resta. En el № 3 se trabajaron definiciones, conceptos y ejercicios contextualizados y en el № 4 se trabajó con ejercicios de aplicación de conocimientos. Talleres 5 y 6 enfocados a la multiplicación. En el taller № 5 se trabajó la parte conceptual y teórica con ejercicios y en el taller №6 se hizo énfasis en la resolución de ejercicios. Talleres 7 y 8 división enfocados a la división. En el taller № 7 se hizo énfasis en conceptos y propiedades y en el taller №8 se realizaron ejercicios de aplicación.

\section{DISCUSIÓN}

Se utilizaron ejemplos de la cotidianidad de los estudiantes para su mejor interpretación y desarrollo, tomando como base las propiedades de cada una de las operaciones, el trabajo se realizó teniendo en cuenta los resultados de las pruebas SABER de los estudiantes durante los dos últimos años, la información fue recogida y analizada mediante la observación directa, evaluación diagnóstica, cuestionario, el diario de campo y prueba de salida y como resultado se encontró que el software Jclic es una herramienta adecuada para fortalecer los procesos de aprendizaje de las operaciones básicas en matemáticas, permitiendo desarrollar problemas contextualizados de una manera diferente y motivando a los estudiantes antes, durante y después del desarrollo de cada actividad, Cabe anotar que el desarrollo de todas las actividades tuvo como base la resolución de problemas; proceso que lleva a los estudiantes a la indagación y exploración, así como a las conclusiones de su propio proceso de aprendizaje. En este sentido, los talleres se desarrollaron en tres fases: Desarrollo de la parte teórica conceptual y ejercicios en hojas de papel, desarrollo del trabajo por medio del software Jclic desde lo teórico y posteriormente desde lo práctico, refuerzo y desarrollo de ejercicios en la casa; se puede afirmar que los estudiantes presentaron una mejoría significativa en el proceso de desarrollo de las operaciones básicas con los números naturales, lo cual, a mediano y largo plazo, ha de redundar en su desempeño académico en el área. 


\section{CONCLUSIONES}

Para establecer las conclusiones, es procedente mencionar el problema a partir del cual surge la investigación, es decir, ¿Cómo fortalecer el desarrollo de las operaciones básicas con los números naturales en los estudiantes del grado tercero en el Centro Educativo Rural Los Guayabales del Municipio de Pamplonita? Se pudieron constatar las siguientes conclusiones:

Se caracterizaron los conocimientos previos sobre las operaciones básicas de números naturales que tienen los estudiantes del grado tercero del Centro Educativo Rural los Guayabales del Municipio de Pamplonita, por medio del №1 "Entrevista a estudiantes" cuyo fin era conocer la percepción de los estudiantes acerca de la forma en que se desarrolla la asignatura de matemáticas y sus motivaciones para tal fin; y por medio del instrumento № 2 "Prueba diagnóstica" con el propósito de verificar el nivel de preparación de los estudiantes para enfrentarse a los objetivos que se espera que logren. De esta manera se logró establecer una serie de deficiencias de las cuales se pueden mencionar las dificultades para sumar y restar llevando, el desconocimiento total o parcial de las tablas de multiplicar, aspectos que dificultan en su totalidad el proceso de la división.

Se diseñó una estrategia didáctica basada en talleres 8 estructurados en 5 momentos que abordaron los diferentes conceptos de las operaciones básicos con los números naturales y utilizando el software JCLIC, teniendo en cuenta el modelo de Aprendizaje Basado en Problemas de las operaciones básicas de los números naturales del grado tercero del Centro Educativo Rural los Guayabales del Municipio de Pamplonita. Cuya intención directa giró en torno a los factores encontrados en la caracterización anterior.

\section{REFERENCIAS BIBLIOGRÁFICAS}

Abrate, Raquel, Pochulu, M., \& Vargas, J. (2006). Errores y dificultades en matemática Análisis de causas y sugerencias de trabajo. Recuperado el 20 de noviembre de 2017, de http://unvm.galeon.com/Libro1.pdf.

Balcázar, P., González, N., \& Gurrola, G. M. (2013). Investigación Cualitativa. Recuperado el 11 de Agosto de 2018, de http://repositorio.minedu. gob. pe/handle/123456789/4641.

Carrillo, S., \& Ríos, J. (13 de marzo de 2009). Trabajo y rendimiento escolar de los estudiantes universitarios. El caso de la Universidad de Guadalajara, México. Recuperado el 20 de Noviembre de 2017, de http://www.scielo. org. mx/pdf/resu/v42n166/v42n166a1.pdf. 
DIGECADE. (2010). Metodología del aprendizaje. Recuperado el 4 de Agosto de 2018, de http://uvg.edu.gt/educacion/maestros-innovadores/documentos/ aprendizaje/ Metodologia.pdf.

Domínguez, L. (2009). Apoyo familiar y desempeño en lengua escrita en escolares de quinto grado de primaria. México.

Educarchile. (2001). Evaluación Incial o Diagnóstica. Recuperado el 11 de Marzo de 2018, de http://ww2.educarchile.cl/User Files/P0001\%5CFile\% 5CEvaluaci\% C3\%B3n\%20 Inicial.pdf.

Elliot, J. (2000). La investigación- acción en educación. Recuperado el 2 de febrero de 2018, de http://www.terras.edu.ar/biblioteca/37/37ELLIOT-Jhon-Cap-1-y-5.pdf.

Euskadi. (2014). Competencia Matemática. Recuperado el 13 de Febrero de 2018, de http://ediagnostikoak.net/edweb/cas/item-liberados/ED09_Euskadi_Matem_EP4.pdf.

Gómez, F. (24 de Julio de 2012). Elementos problemáticos en el proceso de enseñanza de las matemáticas en estudiantes Vicente abadÍA. Recuperado el 23 de Noviembre de 2017, de www.bdigital.unal.edu.co/7052/1/78 10033.2012.pdf.

Hernández, R., Fernández, C., \& Baptista, P. (2010). Metodología de la investigación. México: Editorial Mc Graw Hill.

Instituto Tecnológico de Monterrey. (2002). Recuperado el 29 de noviembre de 2017, de http://sitios.itesm.mx/va/dide2/tecnicas_didacticas/abp/abp.pdf.

Jimeno, M. (2002). Al otro lado de las fronteras de las matemáticas escolares Problemas y dificultades en el aprendizaje matemático de los niños y niñas de tercer ciclo de Primaria. Recuperado el 21 de Noviembre de 2017, de https://riuma.uma.es/xmlui/bitstream/handle/10630/2552/ 16275718.pdf

López, M. (11 de septiembre de 2013). Las nuevas tecnologías en el proceso de enseñanza y aprendizaje. ¿Qué piensan los futuros maestros? Recuperado el 21 de Noviembre de 2017, de https://dialnet.unirioja.es/descarga/articulo/ 4659296.pdf.

López, P. (2004). Población, muestra y muestreo. Recuperado el 11 de agosto de 2018, de http://www.scielo.org.bo/scielo.php?script=sci_arttext\&pid=S1815-02762004000100012.

MEN. (noviembre de 2016). Resumen ejecutivo Colombia en PISA 2015. Recuperado el 21 de noviembre de 2017, de www2.icfes.gov.co/docman/.../2785-informe-resumen-ejecutivocolombia-en-pisa-2016.

MEN. (2018). Resultados nacionales Saber 3oㅗํy yo2012-2017. Recuperado el 8 de Agosto de 2018, de http://www.icfes.gov.co/instituciones-educativas-y-secretarias/pruebas-saber-3-5-y-9/ documentos.

Quecedo, R., \& Castaño, C. (2003). Introducción a la metodología de investigación cualitativa. Recuperado el 11 de Febrero de 2018, de http://www.redalyc. org/pdf/175/17501402.pdf.

Universidad Tecnológica de Pereira. (2017). Diario de Campo. Recuperado el 11 de Marzo de 2018, de https://instrumentos-investigacion.wikispaces. com/4.+ Día rio + de+Campo. 